\title{
Pelvic incidence and pelvic tilt measurements using femoral heads or acetabular domes to identify centers of the hips: comparison of two methods
}

\author{
Marcin Tyrakowski $\cdot$ Hailong Yu $\cdot$ Kris Siemionow
}

Received: 5 August 2014/Revised: 19 December 2014/Accepted: 21 December 2014/Published online: 31 December 2014

(C) The Author(s) 2014. This article is published with open access at Springerlink.com

\begin{abstract}
Purpose The aim of the study was to compare pelvic incidence (PI) and pelvic tilt (PT) measurements using the bicoxofemoral axis obtained from either femoral heads or acetabular domes on lateral digital radiographs of the spine.

Methods Standing lateral radiographs of the spine of patients without hip pathologies were analyzed. PI and PT were measured on 50 radiographs using the femoral heads first followed by measurements performed with the acetabular domes to define the bicoxofemoral axis. Agreement between the methods was quantified by intraclass correlation coefficient (ICC) and median error for a single measurement (SEM). Intraobserver reproducibility and interobserver reliability of both methods of identification of bicoxofemoral axis and its impact on PI and PT measurements were tested on 31 radiographs and quantified by ICC and SEM.

Results There was an excellent agreement in PI as well as in PT between measurements performed using whether the femoral heads or the acetabular domes (ICC: 0.99; SEM: $0.56^{\circ}$ for PI and ICC: 0.99; SEM: $0.2^{\circ}$ for PT). Excellent
\end{abstract}

\footnotetext{
M. Tyrakowski $(\square)$

Department of Orthopedics, Pediatric Orthopedics and Traumatology, The Centre of Postgraduate Medical Education in Warsaw, Konarskiego 13, 05-400 Otwock, Poland e-mail: marcintyrak@gmail.com

M. Tyrakowski $\cdot$ H. Yu $\cdot$ K. Siemionow

Department of Orthopedic Surgery, University of Illinois at Chicago, 835 South Wolcott Ave, Room E-270, Chicago, IL 60612, USA

H. Yu

Department of Orthopedics, General Hospital of Shenyang Military Area Command of Chinese PLA, Shenyang 110016, People's Republic of China
}

intraobserver reproducibility was revealed for both methods (ICC: 0.99 and SEM: $\leq 0.17^{\circ}$ for PI and ICC: 0.99; SEM: $\leq 0.18^{\circ}$ for PT). Both methods presented excellent interobserver reliability (ICC: 0.99 and SEM: $\leq 0.54^{\circ}$ for PI and ICC: $\geq 0.98$; SEM: $\leq 0.9^{\circ}$ for PT).

Conclusions We suggest that either the femoral heads or the acetabular domes may be used for reliable PI and PT measurements on the lateral standing long-cassette digital radiographs of the spine.

Keywords Pelvic incidence - Pelvic tilt · Femoral head . Acetabular dome $\cdot$ Agreement

\section{Introduction}

The double $\mathrm{S}$ shape of the human vertebral column in the sagittal plane is one of the evolutionary adaptations to upright position of the human body, providing a compromise between stability and mobility [1]. Several quantitative parameters, both positional and anatomic, evaluating the spinopelvic sagittal alignment have been reported [2]. Pelvic incidence (PI) first introduced by Duval-Beaupère et al. [3] is the most widely used anatomic parameter. PI is defined as the angle between the line joining the midpoint of the coxofemoral joint axis and the center of the S1 endplate and the line orthogonal to the S1 endplate [3]. Pelvic incidence is hypothesized to be the fundamental pelvic parameter for three-dimensional regulation of spinal sagittal curves [4]. Pelvic tilt (PT), a positional sagittal pelvic parameter, is defined as the angle between the line joining the midpoint of the coxofemoral joint axis to the center of S1 endplate and the vertical reference line [3]. Pelvic tilt revealed to be highly correlated with disability in adult spinal deformity patients $[5,6]$. 
Accuracy of measurements of both, PI and PT, depend on the proper identification of the coxofemoral joints on the lateral long-cassette standing radiograph as well as the S1 endplate. On the traditional X-ray films, the center of each coxofemoral joint was found as the center of the circle surrounding the femoral head. However, some authors used the acetabular dome to find the center of the hip joint [7]. New digital radiographs enable magnification and changes in contrast or brightness of the screen to better visualize the anatomic structures. That is why the acetabular dome and femoral head may be seen as two separate circular lines. According to our clinical observations, femoral heads are more clearly visible on some radiographs, while the acetabular domes on others. Femoral heads and acetabuli have been used interchangeably for PI and PT measurements [811]. In a normal hip joint, a circle drawn on the acetabular dome should be concentric with a circle surrounding the femoral head. Thus, measurements of PI and PT should not be influenced by whether the centers of the femoral heads or the centers of acetabular domes are used for defining the midpoint of the coxofemoral joint axis. However, we do not know if precision of drawing the circles on the borders of the femoral heads or on the acetabular domes on the lateral radiographs is similar, and its influence on PI and PT measurements remains unknown. As to our knowledge, no study that would compare PI and PT measurements using the centers of the femoral heads versus the centers of the acetabular domes to find the midpoint of the coxofemoral axis exists.

The aim of the study was to compare PI and PT measurements performed using either the femoral heads or the acetabular domes to define the center of the coxofemoral joints on standing lateral long-cassette digital radiographs of the spine.

\section{Materials and methods}

\section{Subjects}

After having obtained institutional review board approval, a group of 50 consecutive patients examined between January 2012 and April 2014 with standard standing longcassette postero-anterior and lateral digital radiographs of the spine (General Electric Medical Systems, Centricity PACS Radiology RA1000 Workstation) with clearly visible hip joints, ossified Y cartilage of the acetabulum, ossified proximal femoral physis, and no radiographic signs of hip pathology were retrospectively reviewed and included into the study. There were 27 females and 23 males in mean age of 23 years (14-59 years).

All of the radiographs were downloaded from the Centricity PACS system as bitmap images and analyzed quantitatively in Surgimap Spine Software (Surgimap, New York, USA).

Evaluation of agreement between PI and PT measurements based on femoral heads or acetabular domes to identify the centers of the coxofemoral joints

All of the 50 radiographs were reviewed by a single researcher (orthopedic spine surgeon with 8 years of experience). Two series of measurements were performed at 1-week intervals. The order of radiographs assessed in each series was different and random.

In the first series, PI and PT were measured by use of the "femoral heads method" in the following manner: a line was drawn between the supero-anterior corner and the supero-posterior corner of the S1 vertebral body and was considered as $\mathrm{S} 1$ endplate. Next, a circle was drawn around the borders of each femoral head. The midpoint of the line joining the centers of the circles surrounding the femoral heads (the bicoxofemoral axis) was found automatically by the software. The angle between the line joining the bicoxofemoral axis with the midpoint of the S1 endplate and the line that was perpendicular to the S1 endplate was considered PI and was calculated automatically by the software. Pelvic tilt was defined as the angle between the line joining the bicoxofemoral axis with the midpoint of the $\mathrm{S} 1$ endplate and the reference vertical line and was measured automatically by the software (Fig. 1). After the measurements had been done, the circles were removed from the femoral heads, but the S1 endplate was left in its place and the radiograph was saved in the database.

In the second series of measurements, PI and PT were measured with "acetabular domes method" in the following manner: a line on S1 endplate was saved from the first series of measurements (was exactly the same in both series). Next, a circle was drawn along each acetabular dome instead of the femoral heads (Fig. 2). Pelvic incidence and PT were measured automatically by the software.

Agreement between PI measured with both methods, namely the femoral heads method and the acetabular domes method as well as agreement between PT measured with both methods was tested and quantified by the intraclass correlation coefficient (ICC) and a median error for a single measurement (SEM) [12].

Evaluation of the intraobserver reproducibility for identification of bicoxofemoral axis using the femoral heads or acetabular domes and its impact on PI and PT measurements

In order to assess the intraobserver reproducibility for each method of identification of bicoxofemoral axis and its 


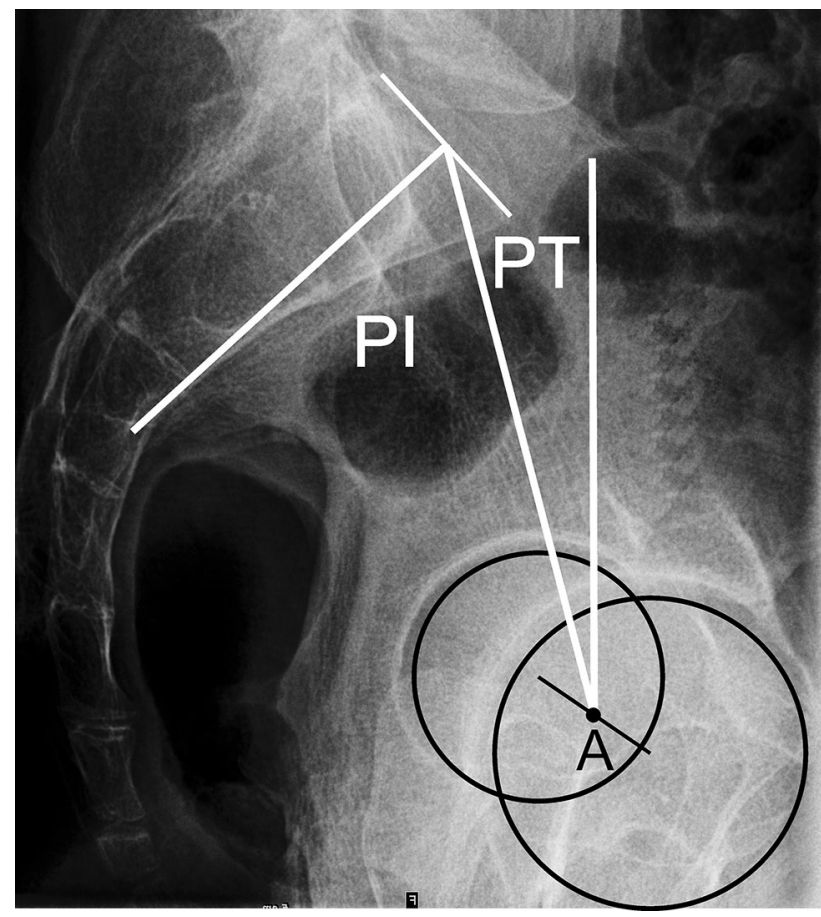

Fig. 1 Pelvic incidence $(P I)$ and pelvic tilt $(P T)$ measured by use of the femoral heads (black circles) to identify the bicoxofemoral axis (A) - "femoral heads method"

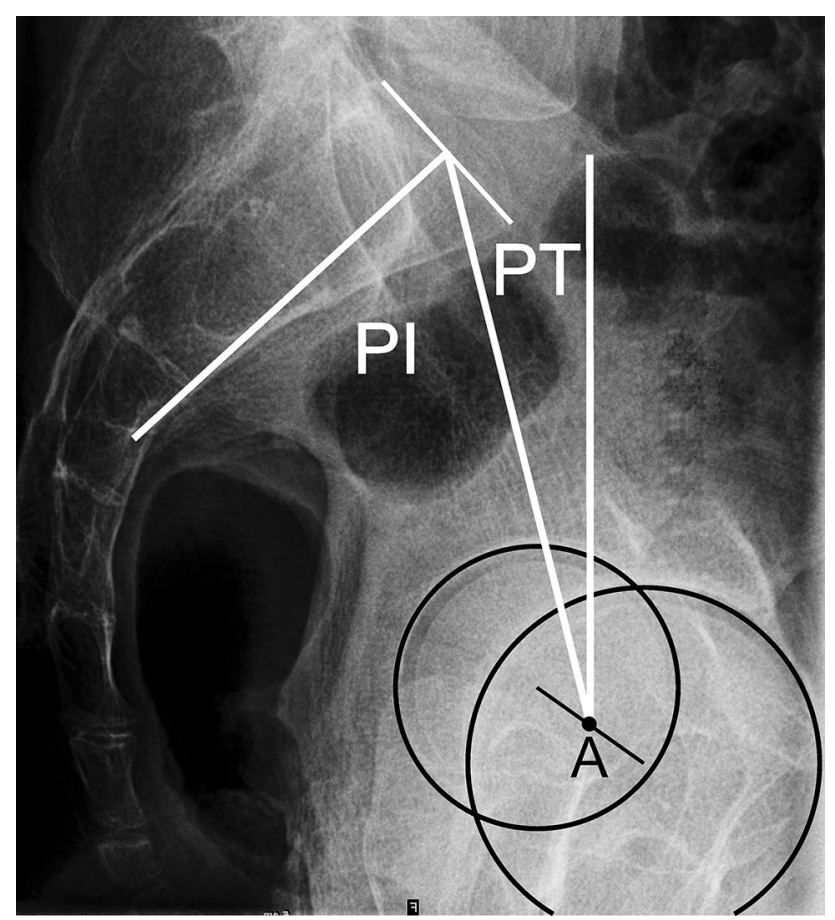

Fig. 2 Pelvic incidence $(P I)$ and pelvic tilt $(P T)$ measured by use of the acetabular domes (black circles) to identify the bicoxofemoral axis $(A)$ - "acetabular domes method"

impact on PI and PT measurements, namely the femoral heads and the acetabular domes method, radiographs of 31 randomly chosen patients were assessed by a single researcher (orthopedic spine surgeon with 8 years of experience). Three series of measurements of PI and PT were performed using the femoral heads method. Next, three series of measurements of the same two pelvic parameters were done on the same radiographs by the same researcher using the acetabular domes method. The line on S1 endplate on a particular radiograph was exactly the same in all of the series of measurements. The order of radiographs in each series was different and random. All of the series of measurements were performed at 1-week intervals. The intraobserver reproducibility for each of both methods of identification of bicoxofemoral axis and subsequently its impact on PI and PT measurements was tested and quantified by ICC and SEM.

Evaluation of the interobserver reliability

for identification of bicoxofemoral axis using the femoral heads or acetabular domes and its impact on PI and PT measurements

Evaluation on interobserver reliability for both methods of identification of bicoxofemoral axis and its impact on PI and PT measurements was performed on the same 31 radiographs that were used for intraobserver testing. Three independent researchers (orthopedic spine surgeons with 5,8 , and 10 years of experience) measured PI and PT first using the "femoral heads method" and followed by the "acetabular domes method." The line on S1 endplate on a particular radiograph was exactly the same in all of the series of measurements, and was predefined by the primary investigator. The order of radiographs assessed by each researcher was different and random. The interobserver reliability for each method of identification of bicoxofemoral axis and its impact on PI and PT measurements was tested and quantified by ICC and SEM.

Statistical analysis

The data were analyzed using the JMP 10.0.2 (SAS Institute Inc, Cary, NC) statistical software and in Microsoft Office Excel 2007 (Microsoft, Redmond, WA).

To estimate the sample size, we treated the ICC value greater than 0.7 (with its $95 \%$ confidence interval of 0.55-0.85) as acceptable agreement/reproducibility/reliability for a research tool [13]. Thus, the minimum number of subjects to test the agreement between two methods of PI and PT measurements was 46 [14]. The minimum number of subjects to test the intraobserver reproducibility (three series of measurements performed by one researcher) as well as the interobserver reliability for three researchers for each method of PI and PT measurements was 31 [14]. 
The ICC value of $<0.40$ indicated poor agreement/ reproducibility/reliability, $0.40-0.75$ indicated fair to good agreement/reproducibility/reliability, and values $>0.75$ reflected excellent agreement/reproducibility/reliability [15].

Randomizations were performed by use of RAND function in Microsoft Office Excel 2007.

\section{Results}

Evaluation of agreement between PI and PT measurements based on femoral heads or acetabular domes to identify the centers of the coxofemoral joints

The mean values of the parameters measured using the femoral heads method were PI: $48.7^{\circ}\left(22^{\circ}-75^{\circ}\right)$ and PT: $10.1^{\circ}\left(-12^{\circ}-30^{\circ}\right)$. The mean values of the parameters measured using the acetabular domes method were PI: $48.9^{\circ}\left(22^{\circ}-75^{\circ}\right)$ and PT: $9.9^{\circ}\left(-12^{\circ}-30^{\circ}\right)$. There was an excellent agreement between the two methods for both PI and PT (Table 1).

Evaluation of the intraobserver reproducibility

for identification of bicoxofemoral axis using the femoral heads or acetabular domes and its impact on PI and PT measurements

There was excellent intraobserver reproducibility of PI and PT measurements performed using whether the femoral heads or acetabular domes to identify the bicoxofemoral axis with ICC of 0.999 and SEM of $<0.2^{\circ}$ for PI and ICC of 0.999 and SEM of $<0.20^{\circ}$ for PT (Table 2).

Evaluation of the interobserver reliability for identification of bicoxofemoral axis using the femoral heads or acetabular domes and its impact on PI and PT measurements

Excellent interobserver reliability of PI and PT measurements performed using whether the femoral heads or acetabular domes to identify the bicoxofemoral axis was revealed with ICC of at least 0.995 and SEM of less than
Table 2 Intraobserver reproducibility for pelvic incidence and pelvic tilt measurements performed with either the "femoral heads method" or the "acetabular domes method"

\begin{tabular}{llllll}
\hline Parameter & \multicolumn{2}{l}{ Femoral heads method } & & \multicolumn{2}{l}{ Acetabular domes method } \\
\cline { 2 - 3 } \cline { 5 - 6 } & ICC & SEM & & ICC & SEM \\
\hline PI & 0.999 & $0.08^{\circ}$ & & 0.999 & $0.17^{\circ}$ \\
PT & 0.999 & $0.18^{\circ}$ & & 0.999 & $0.14^{\circ}$ \\
\hline
\end{tabular}

$P I$ pelvic incidence, $P T$ pelvic tilt, $I C C$ intraclass correlation coefficient, $S E M$ median error for a single measurement

$0.55^{\circ}$ for PI and ICC of at least 0.979 and SEM of $0.90^{\circ}$ or less for PT (Table 3).

\section{Discussion}

We present an analysis comparing two methods of PI and PT measurements on digital lateral radiographs of the spine. Such an analysis has never been published. In this study, both the femoral heads and the acetabular domes were used to identify the bicoxofemoral axis. In the very first study where PI and PT were introduced, DuvalBeaupère et al. [3] used the centers of the coxofemoral joints to define the bicoxofemoral axis. In order to measure pelvic radius, Jackson et al. [7] placed round plastic jar caps with a small hole in their centers just under the superior domes of both acetabula visualized on the lateral radiographs, to mark the centers for the femoral heads. However, subsequent studies specifying normative values of PI and PT as well as their deviations in spinal pathologies used the centers of the femoral heads to define the bicoxofemoral axis [16-23]. The use of acetabula for PI and PT measurements could also be found in the literature [8, 9, 11]. According to our clinical observations, some standing lateral long-cassette radiographs present a more clear view of the femoral heads, while others provide a better visualization of the acetabular domes. That is why we found it relevant to test the agreement between PI and PT measurements using either the femoral heads or the acetabular domes to define the bicoxofemoral axis. In order to investigate if one of the methods is superior to the other

Table 1 Agreement between the method of pelvic incidence and pelvic tilt measurements using the femoral heads ("femoral heads method") or the acetabular domes ("acetabular domes method") to identify the centers of the coxofemoral joints

\begin{tabular}{llrr}
\hline Parameter & Femoral heads method, mean (range) & Acetabular domes method, mean (range) & ICC \\
\hline PI & $48.7^{\circ}\left(22^{\circ}-75^{\circ}\right)$ & $48.9^{\circ}\left(22^{\circ}-75^{\circ}\right)$ & 0.996 \\
PT & $10.1^{\circ}\left(-12^{\circ}-30^{\circ}\right)$ & $9.9^{\circ}\left(-12^{\circ}-30^{\circ}\right)$ & $0.56^{\circ}$ \\
\hline
\end{tabular}

$P I$ pelvic incidence, $P T$ pelvic tilt, $I C C$ intraclass correlation coefficient, SEM median error for a single measurement 
Table 3 Interobserver reliability for pelvic incidence and pelvic tilt measurements performed with either the "femoral heads method" or the "acetabular domes method"

\begin{tabular}{llllll}
\hline Parameter & \multicolumn{2}{l}{ Femoral heads method } & & \multicolumn{2}{l}{ Acetabular domes method } \\
\cline { 2 - 3 } \cline { 5 - 6 } \cline { 5 - 6 } & ICC & SEM & & ICC & SEM \\
\hline PI & 0.995 & $0.54^{\circ}$ & & 0.999 & $0.23^{\circ}$ \\
PT & 0.979 & $0.90^{\circ}$ & & 0.998 & $0.29^{\circ}$ \\
\hline
\end{tabular}

$P I$ pelvic incidence, $P T$ pelvic tilt, $I C C$ intraclass correlation coefficient, $S E M$ median error for a single measurement

intraobserver reproducibility and interobserver reliability of both methods were tested.

The analysis revealed an excellent agreement between the two methods of PI and PT measurements tested. Moreover, both methods presented an excellent intraobserver reproducibility and interobserver reliability in identifying the bicoxofemoral axis and subsequently in PI and PT measurements. Median errors for a single measurement for both methods were negligible with a tendency to be lower for the "acetabular dome method." Our findings suggest that PI and PT may be reliably measured by both methods, and choice of either the femoral heads or the acetabular domes is used to define the bicoxofemoral axis may depend on that which structure is better visible on a particular radiograph or on the physician's preference. We find this as an important clinical implication of the study.

Precision and accuracy of PI measurements were reported by Duval-Baupère et al. [3] to be $2^{\circ}$ and $3^{\circ}$, respectively. Reproducibility for the PI measurements on traditional radiographs was reported to be $6^{\circ}$ [24]. Digital X-rays improved intraobserver reproducibility and interobserver reliability for PI measurements with reported values of $1.1^{\circ}-$ $2^{\circ}$ and $1.5^{\circ}-4^{\circ}$, respectively $[25,26]$. Intraclass correlation coefficient for the intraobserver reproducibility was reported to be 0.69 for PI when manual method was used and up to 0.96 with computer aid $[11,27]$. Our analysis revealed ICC for intra- and interobserver testing of both methods of identifying the bicoxofemoral axis for PI and PT measurements to be $0.98-0.99$ with negligible SEM values. In this study, the line on the S1 endplate was predefined and was the same in all series of measurements. Our findings may therefore indirectly suggest that not defining the bicoxofemoral axis but instead proper positioning of the line on the $\mathrm{S} 1$ endplate is the most important source of inaccuracy in PI and PT measurements. We find this information clinically relevant and suggest that a lot of attention should be given to proper S1 endplate line positioning when PI and PT are measured.

\section{Conclusions}

The study revealed an excellent agreement between the two methods of identifying the bicoxofemoral axis for PI and
PT measurements, namely the method using the femoral heads or the method using the acetabular domes to define the bicoxofemoral axis. Excellent intraobserver reproducibility and interobserver reliability of both methods tested were found. Both the femoral heads and the acetabular domes may be used for reliable PI and PT measurements on the lateral standing long-cassette digital radiographs of the spine. Significant attention should be placed on proper S1 endplate positioning when PI and PT are measured.

\section{Conflict of interest None.}

Open Access This article is distributed under the terms of the Creative Commons Attribution License which permits any use, distribution, and reproduction in any medium, provided the original author(s) and the source are credited.

\section{References}

1. Tardieu C, Bonneau N, Hecquet J, Boulay C, Marty C, Legaye J, Duval-Beaupère G (2013) How is sagittal balance acquired during bipedal gait acquisition? Comparison of neonatal and adult pelves in three dimensions. Evolutionary implications. J Hum Evol 65(2):209-222

2. Vrtovec T, Janssen MM, Likar B, Castelein RM, Viergever MA, Pernuš $F$ (2012) A review of methods for evaluating the quantitative parameters of sagittal pelvic alignment. Spine J 12(5):433-446

3. Duval-Beaupère G, Schmidt C, Cosson P (1992) A Barycentremetric study of the sagittal shape of spine and pelvis: the conditions required for an economic standing position. Ann Biomed Eng 20(4):451-462

4. Legaye J, Duval-Beaupère G, Hecquet J, Marty C (1998) Pelvic incidence: a fundamental pelvic parameter for three-dimensional regulation of spinal sagittal curves. Eur Spine J 7(2):99-103

5. Schwab FJ, Blondel B, Bess S, Hostin R, Shaffrey CI, Smith JS, Boachie-Adjei O, Burton DC, Akbarnia BA, Mundis GM, Ames CP, Kebaish K, Hart RA, Farcy JP, Lafage V, International Spine Study Group (ISSG) (2013) Radiographical spinopelvic parameters and disability in the setting of adult spinal deformity: a prospective multicenter analysis. Spine 38(13):E803-12

6. Schwab F, Patel A, Ungar B, Farcy JP, Lafage V (2010) Adult spinal deformity-postoperative standing imbalance: how much can you tolerate? An overview of key parameters in assessing alignment and planning corrective surgery. Spine 35(25): 2224-2231

7. Jackson RP, Peterson MD, McManus AC, Hales C (1998) Compensatory spinopelvic balance over the hip axis and better reliability in measuring lordosis to the pelvic radius on standing lateral radiographs of adult volunteers and patients. Spine 23(16): 1750-1767

8. Lazennec JY, Brusson A, Rousseau MA (2013) Lumbar-pelvicfemoral balance on sitting and standing lateral radiographs. Orthop Traumatol Surg Res 99(1 Suppl):S87-S103

9. Lazennec JY, Brusson A, Rousseau MA (2012) Hip-spine relations: an innovative paradigm in THR surgery. In: Fokter SK (ed) Recent advances in arthroplasty. In Tech, Rijeka, pp 70-94

10. Le Huec JC, Aunoble S, Philippe L, Nicolas P (2011) Pelvic parameters: origin and significance. Eur Spine J 20(Suppl 5):564-571

11. Peleg S, Dar G, Medlej B, Steinberg N, Masharawi Y, Latimer B, Jellema L, Peled N, Arensburg B, Hershkovitz I (2007) 
Orientation of the human sacrum: anthropological perspectives and methodological approaches. Am J Phys Anthropol 133(3):967-977

12. Shrout PE, Fleiss JL (1979) Intraclass correlations: uses in assessing rater reliability. Psychol Bull 86:420-428

13. Keszei AP, Novak M, Streiner DL (2010) Introduction to health measurement scales. J Psychosom Res 68(4):319-323

14. Zou GY (2012) Sample size formulas for estimating intraclass correlation coefficients with precision and assurance. Stat Med 31(29):3972-3981

15. Streiner DL, Norman GR (2008) Health measurement scales a practical guide to their development and use, vol 4. Oxford University Press, Oxford

16. Barrey C, Jund J, Noseda O, Roussouly P (2007) Sagittal balance of the pelvis-spine complex and lumbar degenerative diseases. A comparative study about 85 cases. Eur Spine J 16(9):1459-1467

17. Boulay C, Tardieu C, Hecquet J, Benaim C, Mouilleseaux B, Marty C, Prat-Pradal D, Legaye J, Duval-Beaupère G, Pélissier J (2006) Sagittal alignment of spine and pelvis regulated by pelvic incidence: standard values and prediction of lordosis. Eur Spine J 15(4):415-422

18. Horduna M, Legaye $\mathbf{J}$ (2008) Influence of the sagittal anatomy of the pelvis on the intercrestal line position. Eur J Anaesthesiol 25(3):200-205

19. Labelle H, Roussouly P, Berthonnaud E, Transfeldt E, O'Brien M, Chopin D, Hresko T, Dimnet J (2004) Spondylolisthesis, pelvic incidence, and spinopelvic balance: a correlation study. Spine 29(18):2049-2054

20. Mac-Thiong JM, Berthonnaud E, Dimar JR 2nd, Betz RR, Labelle $H$ (2004) Sagittal alignment of the spine and pelvis during growth. Spine 29:1642-1647
21. Mac-Thiong JM, Labelle H, Charlebois M, Huot MP, de Guise JA (2003) Sagittal plane analysis of the spine and pelvis in adolescent idiopathic scoliosis according to the coronal curve type. Spine 28:1404-1409

22. Huang RP, Bohlman HH, Thompson GH, Poe-Kochert C (2003) Predictive value of pelvic incidence in progression of spondylolisthesis. Spine 28:2381-2385

23. Roussouly P, Gollogly S, Berthonnaud E, Labelle H, Weidenbaum M (2006) Sagittal alignment of the spine and pelvis in the presence of L5-S1 isthmic lysis and lowgrade spondylolisthesis. Spine 31:2484-2490

24. Lazennec JY, Ramaré S, Arafati N, Laudet CG, Gorin M, Roger B, Hansen S, Saillant G, Maurs L, Trabelsi R (2000) Sagittal alignment in lumbosacral fusion: relations between radiological parameters and pain. Eur Spine J 9(1):47-55

25. Rajnics P, Pomero V, Templier A, Lavaste F, Illes T (2001) Computer-assisted assessment of spinal sagittal plane radiographs. J Spinal Disord 14(2):135-142

26. Boulay C, Tardieu C, Hecquet J, Benaim C, Mitulescu A, Marty C, Prat-Pradal D, Legaye J, Duval-Beaupère G, Pélissier J (2005) Anatomical reliability of two fundamental radiological and clinical pelvic parameters: incidence and thickness. Eur J Orthop Surg Traumatol 15:197-204

27. Dimar JR 2nd, Carreon LY, Labelle H, Djurasovic M, Weidenbaum M, Brown C, Roussouly P (2008) Intra- and inter-observer reliability of determining radiographic sagittal parameters of the spine and pelvis using a manual and a computer-assisted methods. Eur Spine J 17(10):1373-1379 